\title{
Reputation computational model to support electricity market players energy contracts negotiation
}

\author{
Jaime Rodriguez-Fernandez ${ }^{1}$, Tiago Pinto ${ }^{1,2}$, Francisco Silva ${ }^{1}$, Isabel Praça ${ }^{1}$, Zita Vale $^{1}$ and \\ Juan Manuel Corchado ${ }^{2}$ \\ ${ }^{1}$ GECAD - Research group, Institute of Engineering, Polytechnic of Porto (ISEP/IPP), \\ Porto, Portugal \\ \{rfmfa, tmcfp, fspsa, icp, zav\}@isep.ipp.pt \\ ${ }^{2}$ BISITE, University of Salamanca (US), Calle Espejo, 12, 37007 Salamanca, Spain \\ \{tpinto, corchado\}@usal.es
}

\begin{abstract}
The negotiation is one of the most important phase of the process of buying and selling energy in electricity markets. Buyers and sellers know about their own trading behavior or the quality of their products. However, they can also gather data directly or indirectly from them through the exchange information before or during negotiation, even negotiators should also gather information about past behavior of the other parties, such as their trustworthiness and reputation. Hence, in this scope, reputation models play a more important role in decision-making process in the undertaken bilateral negotiation. Since the decision takes into account, not only the potential economic gain for supported player, but also the reliability of the contracts. Therefore, the reputation component represents the level of confidence that the supported player can have on the opponent's service, i.e. in this case, the level of assurance that the opponent will fulfil the conditions established in the contract. This paper proposes a reputation computational model, included in DECON, a decision support system for bilateral contract negotiation, in order to enhance the decision-making process regarding the choice of the most suitable negotiation parties.
\end{abstract}

Keywords: bilateral contracts, decision support system, electricity market, reputation models, negotiation process

\section{Introduction}

Trust and reputation underlies almost every face-to-face trade. The possibility for dealing with strangers significantly increases the risk for such interactions. Hence, relationships based on trust and reputation have been extensively researched the past decade as a result of the huge rise of the virtual communities such as electronic commerce, e.g. $[1,2,3]$. Several research works have conclsuded that seller reputation has significant influences on on-line auction prices $[4,5]$. Trust and reputation have

This work has received funding from the European Union's Horizon 2020 research and innovation programme under the Marie Sklodowska-Curie grant agreement No 641794 (project DREAM-GO) and grant agreement No 703689 (project ADAPT); and from FEDER Funds through COMPETE program and from National Funds through FCT under the project UID/EEA/00760/2013 
become important theme of researcher in many fields. Rather, they group together similar works that have often been of interest to audiences of the various disciplines. In this paper, we focus on how trust and reputation are acquired and used to enhance decision-making process in bilateral negotiation of energy contracts.

The last few decades, energy trade has changed significantly due to the electricity markets (EM) restructuring. Nowadays, several market models exist where each EM has its own characteristics and clearing price mechanisms. This restructuring was promoted by the liberalization and international integration of these markets [6]. Nevertheless, energy trade is essentially supported in all energy markets worldwide by means of bilateral contracts negotiation [7]. The EM liberalization process brings new challenges for the involved entities in the sector, since there are very different types of entities, both at consumers level, which may be smaller or larger; residential, commercial or industrial; urban or rural location, etc.; and at producers level, which may be based on various generation technologies such as thermal energy, cogeneration, or renewable energies (e.g., solar, wind or hydro energy), among others. For this reason, it is essential for the EM participants to have an adequate analysis about the other involved players' behavior with whom they will establish a possible contract.

In is in this scope that trust and reputation play an important role. We focus our attention in computational models that use reputation to model and analyze the agents' trustworthiness throughout information exchange their own interactions. The majority of authors classify reputation from information origin standpoint, both as individual dimension when information is resulting from a direct interaction among agents, and as social dimension when information comes from a community agent that is not participating in current interaction but that has already taken part in past actions with the target agent (witness information) [8]. These systems are usually embedded in intelligent software agents as a mechanism for enabling the agent to make trust-based decisions [9]. Several research analyses of existing online reputation systems such as [10] have analyzed the feedback rating system used in eBay as a reputation system. "Reputation" is taken to be a function of the cumulative positive and non-positive ratings for a seller or buyer $[11,12]$. The REGRET model developed by Sabater and Sierra [12] takes the posture that reputation is composite concept, the overall opinion on an entity is obtained as a result of the combination of different pieces of information.

Using the concept of reputation matured in the literature, and considering the need from energy negotiating players to assess the risk and quality of possible negotiations, this paper proposes a computational reputation model for enhancing support decision during the negotiation of bilateral contracts $[13,14]$. The reputation model endows the proposed decision support methodology with the capability of considering, not only the potential economic gain of the supported player, but also the benefit from a point of view of the contract reliability. The implemented model is based on one of the most recognized reputation models, namely the REGRET model by Sabater and Sierra [15].

\section{Proposed reputation computational model}

Decision making under uncertainty in EM negotiations should take into account, not only the potential economic gain for supported player, but also the reliability of the potential contracts. This way the supported player may choose to undertake 
negotiations with players that present a slightly lower potential profit, but compensate the loss by ensuring safer deals with players that present better reputations, which provide a different level of security, especially regarding the prospect of complying with the terms of the established contract.

The proposed computational reputation model is based on the REGRET model [12] to assess the reputation of bilateral contract negotiating players. The proposed model considers groups according to agents' type. The groups of electric power generators represent the seller players (nuclear, coal, wind, solar, among others) and energy consumer groups represent buyer players (large industries, medium-sized trade, small residential buyers). The supported player can deal both as seller and as buyer, so that players' typology will not be defined in the formulated notation for the model. As follow is presented the reputation computation model:

The reputation of competitor player $p$ is assessed from the perspective of supported player $s p$. It is considered two different components of reputation in order to calculate the reputation of subject competitor player $R_{p}$ :

- The individual component $R_{s p, p}$, which represents the direct observations and experience of the supported player in regard to the subject competitor player;

- The social component $R_{s}$, which considers the perspective of the group in which each player is inserted, and also the society prejudice regarding the player type (e.g., the players who represent the wind farms will trend to own a similar reputation, since they have the same problem to supply a certain amount of energy because they are equally wind speed dependent; on the other hand, regarding the consumers group it is normal the players who belong to large industries have higher reputation than the medium commerce or small players).

$R_{p}$ is, therefore, defined as in (1).

$$
R_{p}=w_{i} \cdot R_{s p, p}+w_{s} \cdot R_{s}
$$

where $w_{i}$ and $w_{s}$ are weights that are attributed to the individual and social component, respectively. The sum of both weights should be equal to 1 , and these should reflect the trust that the supported player has on its own experience and on the experience of others. $R_{s p, p}$ represents a positive or negative experience of the supported player regarding the subject competitor player. This value is updated whenever a new observation is available, and is defined in equation (2).

$$
R_{s p, p}=\frac{N P E}{T N E}
$$

where NPE represents the number of positive experiences and TNE the total number of experiences that the supported player has had with the subject competitor player.

The social component $R_{s}$ allows using information on similar players (e.g. it is unusual that two players of the same group trend to establish different contracts), and also the use of personal experience of the group players belonging the supporter player with the subject player. $R_{S}$ is defined in (3).

$$
R_{s}=w_{g p} R_{s p, G p}+w_{g s p} R_{G s p, p}+w_{g} R_{G s p, g p}+w_{p} P_{s}
$$

Equation 3 shows four different social reputation with their own weights. As follow it will describe each component:

- $R_{s p, G p}$ represents the reputation of the subject competitor player' group from the perspective of the supported player, as defined in equation (4). 


$$
R_{s p, G p}=\sum_{p i \in G p} w_{s p, p i} \cdot R_{s p, p i}
$$

where $\sum_{p i \in G p} w_{s p, p i}=1$. $R_{s p, p i}$ represents the reputation of each member $i$ of the subject competitor player' group (subject competitor player included) from the point of view of the supported player; and $w_{s p, p i}$ represents the weights that are attributed to each these individuals reputations. These weights can be defined according to the credibility of each competitor group from the supported player standpoint.

- $R_{G s p, p}$ represents the reputation of subject competitor player from the perspective the supported player' group, as described in equation (5).

$$
R_{G s p, p}=\sum_{g s p i \in G s p} w_{g s p i, p} \cdot R_{g s p i, p}
$$

where $\sum_{g s p i \in G s p} w_{g s p i, p}=1 . R_{g s p i, p}$ is the reputation of subject competitor player from the point of view of each member $i$ of the supported player' group; and $w_{g s p i, p}$ is the weights that are attributed to each these individuals reputations. These weights can be defined according to the credibility of each competitor group from the supported player standpoint.

- $R_{G s p, g p}$ is the reputation of the subject competitor player' group from the perspective of the supported player' group, as defined in (6).

$$
R_{G s p, g p}=\sum_{g s p i \in G s p} w_{g s p i, G p} \cdot R_{g s p i, G p}
$$

where $\sum_{g s p i \in G s p} w_{g s p i, G p}=1$. $R_{g s p i, G p}$ represents the reputation of each group member where the subject competitor player is inserted (subject competitor player included) from the point of view of each member $i$ of the supported player' group (supported player included); and $w_{g s p i, G p}$ represents the weights that are attributed to each these individuals reputations. These weights can be defined according to the credibility of each competitor group from the supported player standpoint.

- $P_{s}$ represents the preconception of subject competitor player' group. This prejudice value can be attributed by default by an entity recognized as reliable, or as is described in equation (7).

$$
P_{S}=\sum_{g p i \in G p} w_{p i, G p} \cdot R_{p i, G p}
$$

where $\sum_{g p i \in G p} w_{p i, G p}=1 . R_{p i, G p}$ represents the reputation of each members of competitor group from the point of view of each member of opposite group, i.e., whether the supported player is a seller type, it would be the opinion of all generators groups (not only those that the supported player is belonged).

As many notions of reputation have been studied, an intuitive typology of reputation was addressed which was based on the reviewed literature. Figure 1 shows the typology tree of reputation proposed and discussed above.

In order to analyze the responses' trust that are given by each player are defined the different weights that will be attributed to their responses. These weights consider the credibility of the opinions of players. The supported player should be compared the obtained reputation responses to actual experience with the subject player in order to update the related weights and to verify the players' credibility; e.g. if a certain player attributes a large reputation value to the subject competitor player, and when the 
supported player establishes a contract with this opponent verifies that this player is not able to fulfill the contracted conditions, the supported player will not only update the reputation of the competitor player taking into account the bad experience, but will also update the credibility on the responses of the player that provided the misleading evaluation of the competitor player's reputation.

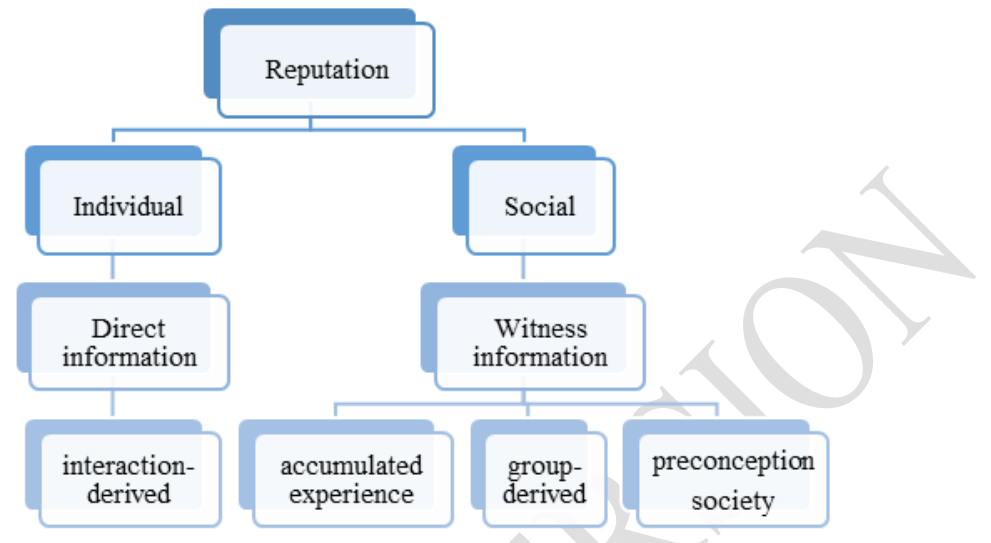

Fig. 1. Proposed reputation typology.

\section{Case study}

\subsection{Specifications}

This section presents an experimental case with the aim of demonstrating the performance of the proposed reputation model to calculate and assess the reputation values of each subject competitor in order to be used to support the bilateral negotiation decisions in electricity market.

The study contemplates a single scenario with both player types. Energy generator or sellers, and consumers or buyers, where in turn each player is related in a group. Hence, this simulation case involves 5 consumers and 4 generators, where the supported player is a seller player. As follow it is defined the related group to each player:

- Consumers (3 groups):

Small player $\rightarrow$ Buyer 1

Medium commerce $\rightarrow$ Buyer2, Buyer5

Large industry $\rightarrow$ Buyer3, Buyer4

- Generators (2 groups)

Wind farm $\rightarrow$ Seller3 (the supported player), Seller4

Solar power plant $\rightarrow$ Seller1, Seller2

As detailed below the Table 1 presents the individual reputation values of the all buyers from the personal experience of each seller. It should be noted that no realistic 
reputation values referent of each competitor player, since it would require a sociologic study, which is out of the scope of this work. Hence, all reputation values were normalized ranging from 0 to 1 , and it has been assumed reasonable default reputation values which were attributed to each player according to the group type belonging; e.g., if a buyer is inserted in the large industry group will have a high reputation value from the point of view of the sellers between 0.7 and 1 , a medium commerce buyer will own a reputation value between 0.4 and 0.6 , and a small buyer normally has a low reputation value from the perspective of sellers between 0 and 0.3 .

TABLE I. Buyers reputation network from the sellers' standpoint.

\begin{tabular}{|c|ccccc|}
\cline { 2 - 6 } \multicolumn{1}{c|}{} & Buyer1 & Buyer2 & Buyer3 & Buyer4 & Buyer5 \\
\hline Seller1 & 0.3 & 0.4 & 0.8 & 0.9 & 0.5 \\
\hline Seller2 & 0.2 & 0.4 & 1.0 & 0.7 & 0.5 \\
\hline Seller3 & $\mathbf{0 . 8}$ & $\mathbf{0 . 3}$ & $\mathbf{0 . 1}$ & $\mathbf{0 . 1}$ & $\mathbf{0 . 3}$ \\
\hline Seller4 & 0.0 & 0.6 & 0.7 & 1.0 & 0.4 \\
\hline
\end{tabular}

Table I shows blue light shaded cells that difference the supported player' group of other seller groups. In order to validate the influence of the personal experience of the supported player (seller3) for the reputation calculation, it can observe in Table I that it has supposed an opposite scenario in relation to other sellers, i.e. it has not considered reasonable reputation values, it is just that it has assumed high values of reputation in regard to small buyers and low values to large and medium buyers. This will allow an easier verification of the influence of the individual component on the proposed reputation model.

On the other hand, it will be analyzed the influence of the weights for each component. For that purpose, it has defined three simulation cases considering different weight values for the components, as both the individual component $w_{i}$ as the social component $w_{s}$. For the Case 1 , it is defined $w_{i}=0.5$ and $w_{s}=0.5$; in the Case $2, w_{i}=0.8$ and $w_{s}=0.2$; and finally the Case 3 , it is considered $w_{i}=0.2$ and $w_{s}=0.8$.

Hence, the overall goal is to allow a great analysis of the results provided by the reputation model to assess the reputation of subject competitor players, and how these results can change according to the credibility that is given to each player by means of the weights.

\subsection{Results}

Executing the implemented reputation model according to the previous specifications, it has been obtained the work results which will allow to assess the reputation of subject competitor players for enhancing the decision-making process of bilateral contract negotiations in electricity markets.

The following table shows the reputation result of each subject competitor player for the different cases of weights allocation that was previously mentioned, as well as the related reputation with each component. As previously was explained with detail in the Section III, the individual component will represent the personal experience of the supported player between the various subject competitors; and the social component 
will represent the perspective of the supported player' group regarding their opinion about subject competitor players.

TABLE II. Reputation of the subject competitor players considering different cases of weights allocation.

\begin{tabular}{lccccc}
\hline \multicolumn{1}{c}{ Buyer ID } & $\mathbf{1}$ & $\mathbf{2}$ & $\mathbf{3}$ & $\mathbf{4}$ & $\mathbf{5}$ \\
\hline $\begin{array}{l}\text { Individual Component } \\
\text { Social Component }\end{array}$ & 0.80 & 0.30 & 0.10 & 0.10 & 0.30 \\
\hline Reputation Case 1 & 0.36 & 0.48 & 0.57 & 0.64 & 0.43 \\
Reputation Case 2 & 0.58 & 0.39 & 0.33 & 0.37 & 0.37 \\
Reputation Case 3 & 0.71 & 0.34 & 0.19 & 0.21 & 0.33 \\
\hline \hline
\end{tabular}

Observing the Table II it can affirm that the weights distribution has influence on the total reputation, since the social opinion about the negotiators targets does not coincide with the individual opinion of supported player.

Looking detailed to the different simulation cases, in Case 1 all reputation values are similar among the various buyers, since as the weights of social and individual components are considered equals, the contradictory opinions between the supported player and the other sellers enabling that reputations are balanced; and in this way, none of the opinions are favored. In Case 2, it is a clear example of the influence of the supported player opinion which has a greater weight for the reputation calculation, and thus, the small buyer (Buyer1) has a rather large value, and the large and medium buyers present strange reputation, much lower in comparison with the values considered as usual. The latter simulation, the Case 3 considers the smallest influence of the supported player, and reputations assume more similar values to the usual ones, i.e. large buyers (Buyer3 and Buyer4) have the highest reputation values, although the small buyer reputation is still favored by the hard influence of the supported player opinion.

In summary, this proposed scenario where the opinion of the supported player in relation to the others sellers is contrary, is an easy scenario for showing the influence of the different weights for the total reputation calculation. In this way, the reputation of the small competitors is favored, however the group of large and medium buyers present a worst reputation in comparison with reputations consider as usual (low values for small consumers, middle values for consumers of size medium and high values for large consumers). Hence, it can conclude that the obtained results provided by the proposed reputation model are according to the expected.

\section{Conclusions}

Pre-negotiation is a process that assumes great relevance in bilateral negotiations, as it is the phase when all the preparation and planning of the effective negotiation in energy contract negotiation is carried out. In this process, the purpose is to identify the ideal negotiators to approach the negotiations, in order to obtain the maximum possible 
benefit for the supported player. Therefore, an important factor in the choice of the opponents to negotiate is to take into account the ability and reputation of the latter to be able to comply with the contract. For this reason, it is crucial to develop ways for analyzing and modeling the reputation and credibility of the different potential negotiators, so that the decision-making process can be improved.

A reputation model has been proposed in this paper, showing the influence of the different weights of the social and individual components through the various simulation cases. The influence of different opinions that the supported player can present about the target negotiators has also been assessed. It can then be concluded that the results obtained by the proposed model correspond entirely to what would be expected, being therefore the model validated as fulfilling its purpose to enhance the decision support process for EM players by means of bilateral contract negotiation.

As future work, a model for the credibility of the involved players is proposed. Since the reputation model needs reputational values that are suggested by other players, these opinions may not be entirely reliable because opinions are being asked from negotiating agents, which can create a conflict of interest. A model capable of evaluating the credibility of the different players' responses would allow the automatic adaptation of the weights assigned to the responses of each player according to their credibility.

\section{References}

1. H. Rheingold (2001) The Virtual Community: Homesteading on the Electronic Frontier, Revised Edition. Cambridge, MA: MIT Press.

2. $\quad$ B. Wellman (2001) "Computer Networks As Social Networks." Science 293(14), pp. 2031-2034.

3. M. Smith and P. Kollock (eds., 1999) Communities in Cyberspace. London: Routledge Press.

4. D. E. Houser and J. Wooders (2001) "Reputation in Internet Auctions: Theory and Evidence from eBay." working paper: http://w3.arizona.edu/ econ/working_papers/Internet_Auctions.pdf.

5. S. Dewan, V. Hsu (2001) "Trust in Electronic Markets: Price Discovery in Generalist Versus Specialty Online Auctions." http://databases.si.umich.edu/reputations/bib/papers/Dewan\&Hsu.doc.

6. Sioshansi, F.P., "Evolution of Global Electricity Markets - New paradigms, new challenges, new approaches", Academic Press, 2013.

7. Algarvio, H.; Lopes, F.; Santana, J. Bilateral Contracting in Multi-agent Energy Markets: Forward Contracts and Risk Management. Highlights of Practical Applications of Agents, Multi-Agent Systems, and Sustainability-, Springer International Publishing 2015, 260- 269.

8. A. Gutowska, K. Buckley. 2008. "Computing Reputation Metric in Multi-Agent E-Commerce Reputation System". International Conference on Distributed Computing Systems, Beijing.

9. J. Sabater, C. Sierra. 2005. "Review on computational trust and reputation models". Artificial Intelligence Review.

10. P. Resnick, R. Zeckhauser (2000) "Trust Among Strangers in Internet Transactions: Empirical Analysis of eBay's Reputatoin System." NBER Workshop on Empirical Studies of Electronic Commerce Paper.

11. P. Resnick , K. Kuwabara, R. Zeckhauser , E. Friedman (2000a) "Reputation Systems." Communications of the ACM, 43(12): 45-48.

12. J. Sabater, C. Sierra. 2001. "REGREAT: A reputation model for gregarious societies". A reputation model for gregarious societies.

13. T. Pinto. 2016. "Decision Support for the Strategic Behaviour of Electricity Market Players". Doctorate Degree. University of Trás-os-Montes e Alto Douro.

14. T. Pinto, Z. Vale, I. Praça, E.J. Solteiro, F. Lopes. 2015. "Decision Support for Energy Contracts Negotiation with Game Theory and Adaptive Learning". Energies

15. Sabater, J.; Sierra, C. Review on computational trust and reputation models. Artificial Intelligence Review, 2005, 24, 1, $33-60$. 